\title{
Análisis ambiental debido a la transición energética de la cascarilla de café: caso de estudio en el municipio de Villanueva, La Guajira
}

Daniela Torres', Marlon Bastidas

\section{Resumen}

En este trabajo se analizaron las propiedades de la cascarilla de café como fuente sustentable de biomasa en el municipio de Villanueva, La Guajira, teniendo en cuenta que es uno de los principales productos cultivados en la región. Esta revisión se realizó mediante fuentes bibliográficas enfocadas en las temáticas de la cascarilla de café, el impacto ambiental generado por las cascarillas y las diferentes metodologías empleadas en la transición energética de este residuo con la finalidad del establecimiento de datos en los que se emplee este proceso para que generare valor agregado a un residuo que pasa a ser producto final, de manera que se vayan disminuyendo los impactos ambientales negativos e impulsando el adecuado manejo, aprovechamiento y disposición del residuo sólido. El estudio demostró que la cascarilla de café es una fuente primordial de biomasa energética, por lo que se concluye que es una alternativa sostenible de alto poder calorífico y que puede ser empleada por su eficiencia energética como una estrategia en la disminución potencial de los efectos negativos en los recursos naturales impactados.

Palabras clave: aprovechamiento, beneficio del café, transición energética, biomasa, biocombustibles, subproductos, impactos ambientales. 


\section{Introducción}

A diario, la generación de residuos agrícolas va en aumento, originando problemáticas ambientales como la contaminación de los recursos naturales, contaminación visual, entre otros, debido a la inadecuada disposición que se realiza en las áreas del cultivo. Sinergia (2006) afirma que "la agricultura se puede definir como la actividad que ejerce el hombre haciendo uso deliberado de la tierra para extraer bienes del suelo gracias al aprovechamiento de la energía solar. Es una actividad estratégica para cualquier sociedad". Sin embargo, en los países productores de café, tales como Brasil, Vietnam y Colombia, los residuos y subproductos constituyen una fuente grave de contaminación; en algunos casos, en países con producción a menor escala como Costa Rica, este producto ha sido señalado como responsable de numerosas complicaciones ambientales, reproches y cuestionamientos por los que se paga un elevado precio. Así mismo, las investigaciones sobre el tema solo se han dirigido a mejorar la productividad cafetalera, dejando al margen la consideración de sus repercusiones ambientales (Granados, 1994).

Varios estudios a nivel mundial han informado que los residuos sin tratar, procedentes de las industrias tradicionales y modernas de procesamiento de café, están amenazando las aguas superficiales, mayoritariamente en los países en desarrollo, siendo la contaminación del agua el de mayor repercusión en los países productores de café (Beyene et al., 2012). En Brasil, la producción en los últimos cinco años varió de 2,0 a 2,7 millones de toneladas, dicha producción representa un promedio de más de 2,5 millones de toneladas de residuos sólidos que se generan cada año (Oliveira et al., 2008).

En Colombia existen alrededor de 955000 hectáreas de café a lo largo y ancho del país, ubicadas entre los 1100 msnm y los 2000 msnm en 21 departamentos y 592 municipios, dentro de las cuales La Guajira reflejó en producción 3924 toneladas de café en el 2014 (MADR, 2014). En el procesamiento del café se estima que menos del $5 \%$ de la biomasa generada se aprovecha en la elaboración de la bebida aromática, el resto queda en forma residual, representado en materiales lignocelulósicos como cáscaras, pulpa de café, tallos y hojas, los cuales influyen en la contaminación del medio ambiente por no ser tratados o reciclados (Rodríguez y Zambrano, 2010). 
El método de procesamiento de café húmedo que se utiliza comúnmente requiere enormes cantidades de agua para quitar la cáscara de café en donde se generan volúmenes de aguas residuales con alto contenido en materia orgánica. El efluente de una tonelada de café siguiendo el proceso de beneficiado húmedo genera una demanda bioquímica de oxígeno comparable a la DBO de los residuos humanos que puede ser generada por 2000 personas al día. (Beyene et al., 2012)

Puesto que los desechos del proceso de beneficiado húmedo del café se consideran como contaminantes, el caficultor debería tener una alternativa de uso de estos residuos orgánicos, ya que en la actualidad ha incrementado el interés en buscar alternativas que permitan una disposición adecuada de los residuos y un aprovechamiento de los mismos, por ejemplo, la transición energética.

Así pues, la cascarilla de café puede ser utilizada en diferentes procesos debido a sus propiedades. Un ejemplo de esto fue lo propuesto por Barón (2014), que empleó como material adsorbente para la remoción de iones $\mathrm{Pb} 2+$ presente en soluciones acuosas, presentando una capacidad máxima de adsorción de 4,80 $m g g-1$.

De otra manera, Romero y Mamani Pari (2013)

utilizaron biodigestores a condiciones meteorológicas del valle Yanatile, Cusco con el objetivo de obtener biogás y el residuo del proceso (lodo) como bioabono, obteniendo 6430912,6 m3 de biogás como estimado, compuesto por: $65,2 \%$ de metano, $29,7 \%$ de bióxido de carbono y $5 \%$ (otros)

Así mismo, Carrillo et al. (2011)

planteó la obtención de biomasa a partir de cáscara de café en donde la cáscara de café constituyó un sustrato adecuado para la producción de biomasa con un rendimiento de 3,83 $\mathrm{g} / \mathrm{L}$ de biomasa, cuyo contenido de proteínas fue de $41,49 \%, 16,51 \%$ de azúcares totales, 4,07\% de lípidos y $15,99 \%$ de humedad.

Por otro lado, la cascarilla de café representa una afectación de gran importancia, teniendo en cuenta los impactos ambientales que el proceso de beneficiado húmedo del café representa para los sistemas acuáticos, por lo que Louzada, Andrade-Vieira et al. (2016) evaluaron 
el potencial fitotóxico, citogénico y mutagénico del agua residual del café en la germinación de semillas, elongación de la raíz y alteraciones del ciclo celular en el modelo de planta Lactuca sativa L., en el que la germinación total de las semillas estuvo inhibida después de la exposición al efluente sin tratar. Igualmente, Beyene et al. (2012) realizaron una evaluación de la calidad del agua en la que los análisis fisicoquímicos biológicos revelaron el alto deterioro de la calidad en aguas abajo, encontrándose la carga orgánica más alta de 1900 mg/L, el oxígeno disuelto disminuyó a $0,01 \mathrm{mg} / \mathrm{L}$, reduciéndose de esta manera la nitrificación, y la cantidad de los macroinvertebrados descendió.

El municipio de Villanueva, La Guajira, no escapa a toda la problemática planteada en los párrafos anteriores, pues la siembra de café es una de las principales características de esta zona, en la cual no existen medidas que direccionen a la recolección y aprovechamiento de los residuos generados en la labranza, siendo los recursos naturales los más afectados, ya que si estos no se tratan adecuadamente ocasionan focos de contaminación tanto en acuíferos como en el suelo; en algunos casos, como acciones de vertidos de residuos o su quema a cielo abierto, no son considerados como un problema pues esta costumbre viene instaurada desde hace mucho tiempo, ya que la falta de conocimiento o compromiso incita a los agricultores a no aceptar soluciones consecuentes, sobre todo cuando lo intervenido se relaciona directamente con su sustento económico (Cubides y Montaña, 2017).

Con esta revisión bibliográfica, se nota que los estudios están encaminados al análisis de la cascarilla de café, en los que se analizan los impactos ambientales causados y la posibilidad energética que presenta dicho residuo; en este trabajo se recogen los resultados más importantes, presentando una información general de la situación que permite la comparación y extrapolación a nuevos escenarios como el del aprovechamiento en la agricultura de la cascarilla de café en el municipio de Villanueva, La Guajira, empleando la transición energética del residuo.

\section{Metodología}

Se realizó una revisión bibliográfica en bases de datos reconocidas como ScienceDirect, Elsevier, ResearchGate y Dialnet, en las que se realizó un barrido de trabajos en un 56,8\% de artículos entre el 2014 al 2018, 21,5\% de 2010 a 2013 y un $21,5 \%$ con fecha menor al 2010, con un total de 51 artículos. 
Con la información obtenida se hizo una clasificación de temáticas directamente relacionadas a la cascarilla de café, el impacto ambiental generado por las cascarillas y las diferentes metodologías empleadas en la transición energética de este residuo agrícola. Esta información se utilizó para la conformación de datos en la zona rural proyectada del municipio de Villanueva.

Este municipio se encuentra ubicado al sur del departamento de La Guajira (Colombia) el cual limita con los municipios del Molino por el Norte, Urumita al Sur, San Juan del Cesar y el Departamento del Cesar al occidente y con la República de Venezuela al oriente, el municipio de Villanueva se encuentra situado geográficamente en las coordenadas $10^{\circ} 36^{\prime} 19^{\prime \prime}$ de latitud Norte y $72^{\circ}$ $58^{\prime} 48^{\prime \prime}$ de longitud Oeste como se observa en la figura 1 . Se encuentra concertado en la zona urbana, pero su actividad económica está centrada en la agricultura y ganadería. La extensión del municipio es de 26770 ha, de las cuales 7300 ha son utilizadas para la agricultura y 8600 ha para la ganadería; 10416 son áreas no cultivadas mientras 454 corres- ponden al área urbana. (Municipio de Villanueva, 2000)

El café ha sido uno de los cultivos más tradicionales pues desde tiempos inmemoriales se ha visto que utilizan las calles y parques para el secado de este, incluso Villanueva se encuentra dentro del Comité de cafeteros del Cesar y la Guajira el cual regula a cerca de 250 caficultores y proporciona la venta del grano a distintas federaciones. Personalmente y con los documentos adecuados se fue a solicitar la información de hectáreas sembradas, producción y los procesos realizados en la cosecha y a parte que utilidades les ofrecen a los subproductos del café en especial a la cascarilla, pero fue imposible recibir respuesta alguna. Ante esta negativa se consultó en la página del Ministerio de Agricultura y Desarrollo Rural y se pudo conocer que en año 2014 en la Guajira el área sembrada del café fue de 6790 ha, área cosechada de 6079 ha con una producción de 3924 ha con un rendimiento de 0,65 (t/ha). (MADR, 2014)

Así mismo, para Villanueva se obtienen los datos que se indican en la tabla 1 . 
Tabla 1. Área sembrada, área cosechada, producción y rendimiento del cultivo de café en Villanueva, 2014

\begin{tabular}{|l|l|l|l|l|}
\hline & $\begin{array}{l}\text { Área sembrada } \\
\text { (ha) }\end{array}$ & Área cosechada (ha) & Producción (t) & $\begin{array}{l}\text { Rendimiento } \\
\text { (t/ha) }\end{array}$ \\
\hline Café & 1.167 & 1.028 & 734 & 0.7 \\
\hline
\end{tabular}

Fuente: MADR (2014).

Figura 1. Localización del caso de estudio: municipio de Villanueva, La Guajira

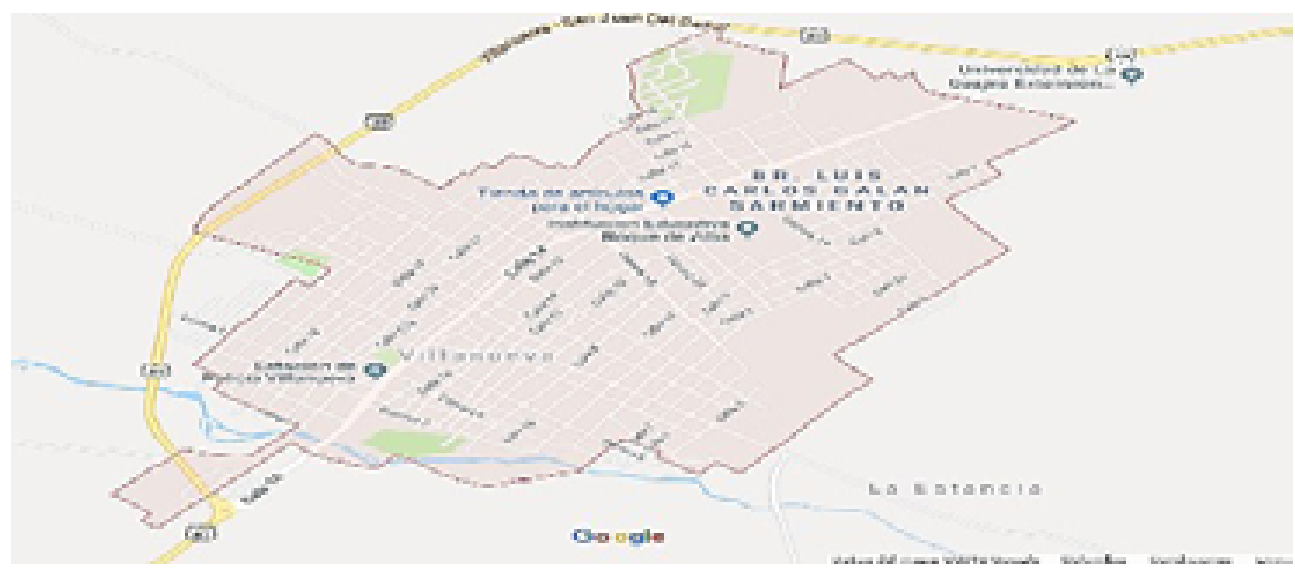

Fuente: Google Maps.

\section{Discusión de resultados}

Por medio del barrido de artículos se realizó una clasificación por año, en la que se da a conocer la temporada en que se enfatizó en la temática asociada a la cascarilla de café. En la figura 2 se puede observar que entre 2010 al 2013 y con fecha menor al 2010 solo el $21,5 \%$ de artículos desarrollaron este tema mien- tras que del 2014 al 2018 se encontraron cerca de $56,8 \%$, obteniendo el mayor porcentaje, esto podría estar sujeto a la concientización de los últimos años sobre el uso indiscriminado de los combustibles fósiles y a la preocupación de emplear tecnologías amigables con el medio ambiente. 
Figura 2. Clasificación de los artículos revisados por años

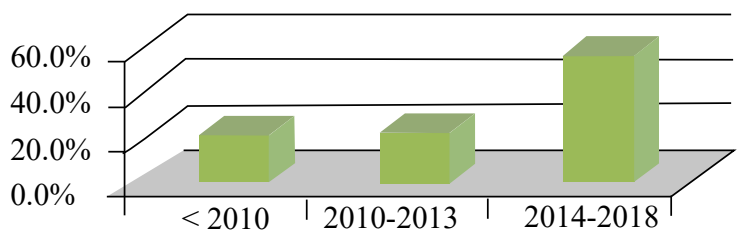

De otra forma, se identificaron los autores que constantemente publican artículos acerca de la cascarilla de café como se observa en la tabla 2, siendo recurrentes Jofran Luiz de Oliveira, Jadir Nogueira da Silvab, Emanuele Graciosa Pereira con la publicación de dos artículos en el 2013 (De Oliveira et al., 2013) y (De Oliveira et al., 2018) en el 2018. De igual manera, Leandro S. Oliveira, Adriana S. Franca y Sonia D. Rocha con la publicación de tres artículos en el 2007 (Oliveira et al., 2008), en 2008 (Oliveira et al., 2008) y en el 2009 (Franca y Oliveira, 2009).

Tabla 2. Identificación de autores recurrentes en la temática de cascarilla de café

\begin{tabular}{|c|c|c|}
\hline Años & Autores & $\begin{array}{l}\text { Número de } \\
\text { artículos }\end{array}$ \\
\hline \multirow{3}{*}{2013 y 2018} & Jofran Luiz de Oliveira & \multirow{3}{*}{2} \\
\hline & Jadir Nogueira da Silva & \\
\hline & $\begin{array}{l}\text { Emanuele Graciosa } \\
\text { Pereira }\end{array}$ & \\
\hline \multirow{3}{*}{$\begin{array}{l}\text { 2007, } 2008 \text { y } \\
2009\end{array}$} & Leandro S. Oliveira & \multirow{3}{*}{3} \\
\hline & Adriana S. Franca & \\
\hline & Sonia D. Rocha & \\
\hline
\end{tabular}

Figura 3. Temáticas principales analizadas en la revisión bibliográfica realizada

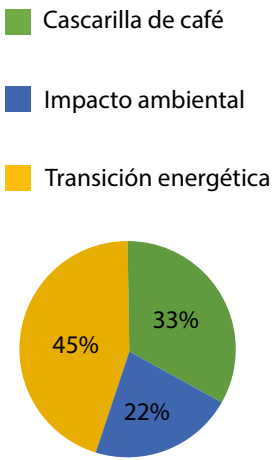

Así mismo, en la figura 3 se puede observar que de los 51 artículos analizados el $45 \%$ corresponde a la temática de transición energética, en la que se 
emplean diferentes metodologías para el aprovechamiento de la cascarilla de café, el $33 \%$ corresponde a artículos enfocados a la caracterización de la cascarilla los que se dan a conocer propiedades físicas, químicas y calóricas que la permiten ser considerada como biomasa y utilizar sus características para diferentes aplicaciones comunes y el $22 \%$ restante corresponde a artículos en los que se dan a conocer metodologías y alternativas enfocadas a contrarrestar los efectos negativos producto del manejo y disposición inadecuada de la cascarilla de café por parte de los agricultores e industrias especializadas en el tema.

\section{Análisis de temáticas}

La cascarilla de café. La caracterización fisicoquímica y calórica de la cascarilla de café es de especial importancia, pues con base en esto se puede conocer si este residuo puede ser considerado como biomasa. En la tabla 3 se realizó una comparación de datos de diversos autores, la que se destacan y comparan diferentes parámetros del residuo. De acuerdo con Toscano (2009) y Arias y Meneses (2016), el poder calorífico de la cascarilla se encuentra entre 14215 $\mathrm{kJ} / \mathrm{kg}$ y $31204 \mathrm{~kJ} / \mathrm{kg}$, respectivamente. Estos valores, con tan notable diferencia, pueden deberse al tipo de café o al lugar donde es cultivado, teniendo en cuenta que estos autores realizaron la investigación en Guayaquil, Ecuador, donde se siembra arábigo lavado, arábigo natural y robusta y en Managua, Nicaragua, la variedad arábiga. Con respecto al porcentaje de humedad, se observa que se encuentra en un promedio de $10,6 \%$, siendo el valor mínimo reportado entre $8,6 \%$ y $9,3 \%$. Cabe mencionar que para procesos energéticos la biomasa debe de tener un contenido de humedad inferior al 30\%. El material volátil y el diámetro de la partícula son directamente proporcionales, pues al disminuir el tamaño de la partícula la cantidad de volátiles se reduce y, además, la ceniza aumenta como lo reportado por Arias y Meneses (2016) y Manals, Salas y Penedo (2018). Igualmente, en la misma tabla, como parte del análisis último realizado en Toscano (2009), Manals, Salas y Penedo (2018) y Conesa et al (2016) se destaca que los valores estuvieron en el rango establecido por los autores expuestos; en este apartado no hubo mucha diferencia entre estos. Entre otros elementos componentes de la cascarilla se encuentran el azufre con un porcentaje mínimo de $0,3 \%$ $\mathrm{y}$ el fósforo y el potasio con $0,07 \% \mathrm{y}$ $2,67 \%$, respectivamente. El calcio con un porcentaje superior de 3,01\% y magnesio $0,33 \%$ (Conesa et al., 2016). De 
ahí que los metales alcalinos y alcalinotérreos abunden en las cenizas de cáscara de café, estas podrían ser adecuadas para reemplazar a los feldespatos tradicionales que se usan mayoritariamente como fundentes en formulaciones cerámicas a base de arcilla (Acchar, Dultra y Segadães, 2013). Por otra parte, el porcentaje de celulosa, hemicelulosa y lignina observada en la tabla 2 reportado por Arias y Meneses (2016) y Álvarez (2016) difieren alrededor de 12\%, 19\% y $7 \%$, respectivamente. Estas diferencias son atribuibles a la diversidad del café cultivado; sin embargo, los valores obtenidos son ideales para la obtención de azúcares más simples y bioetanol a través de la transformación de la celulosa (Arias y Meneses, 2016).

Tabla 3. Caracterización fisicoquímica y calórica de la cascarilla de café según diversos autores

\begin{tabular}{|l|c|c|c|c|c|}
\hline \multicolumn{5}{|c|}{ Caracterización fisicoquímica y calórica de la cascarilla de café } \\
\hline Parámetros & {$[16]$} & {$[17]$} & {$[18]$} & {$[19]$} & {$[21]$} \\
\hline Poder calorífico (kJ/kg) & 14215 & & & & \\
\hline Contenido de humedad (\%) & 10 & 10,1 & 8,6 & 15 & 9,3 \\
\hline Material volátil (\%) & - & 82 & 87,7 & - & - \\
\hline Contenido de cenizas (\%) & 0,6 & 1,2 & 10,5 & 5,4 & 3,2 \\
\hline Carbono (\%) & 46,4 & 50,3 & - & - & 44 \\
\hline Hidrógeno (\%) & 4,86 & 5,3 & - & - & 6,1 \\
\hline Oxígeno (\%) & 46,7 & 43,8 & - & - & 45,3 \\
\hline Nitrógeno (\%) & 0,59 & $<$ & - & - & 1,1 \\
\hline Azufre (\%) & 0,59 & - & - & - & 0,3 \\
\hline Fósforo (\%) & - & - & 0,1 & - & 0,07 \\
\hline Potasio (\%) & - & - & 3,03 & - & 2,67 \\
\hline Calcio (\%) & - & - & 0,25 & - & 3,01 \\
\hline Magnesio (\%) & - & - & 0,05 & - & 0,33 \\
\hline Celulosa (\%) & - & - & 36,7 & 24,5 & - \\
\hline Lignina (\%) & - & - & 15,93 & 23,7 & - \\
\hline Hemicelulosa (\%) & - & - & 47,37 & 27,7 & - \\
\hline
\end{tabular}


Hay que mencionar, además, que la cascarilla de café tiene diversas aplicaciones, las cuales pueden observarse en la figura 4. "Entre estas, se utiliza la cascarilla como sustrato en el crecimiento de la levadura Candida utilis con un rendimiento de $3,83 \mathrm{~g} / \mathrm{L}$ de biomasa, proteínas $41,49 \%, 16,51 \%$ de azúcares totales, 4,07\% lípidos y 15,99\% de humedad" (Carrillo et al., 2011). Como materia prima según Roque, Medrano et al (2013) en la elaboración de materiales de construcción con una proporción de cascarilla del 10\%; de igual forma, Coffea (2010) en la elaboración de papel a partir de la cascarilla debido al porcentaje de celulosa de la cascarilla de café (24,5\% y 36,7\%), principal componente del papel, y Acchar, Dultra et al. (2013), quienes utilizaron las cenizas de la cáscara de café como fundente en baldosas de cerámica. Por último, utilizadas como material biosorbente para el tratamiento de aguas contaminadas con colorantes (Oliveira et al., 2008). Así mismo, la cascarilla de café es usada con total eficacia para la remoción de iones de $\mathrm{Pb2+}$, en los que la capacidad de adsorción máxima fue de $37,04 \mathrm{mg} / \mathrm{g}$ en soluciones acuosas (Alhogbi, 2017). Entre otros usos, se destaca la utilización de esta en la erosión del suelo y escorrentía. En los resultados obtenidos se observó que los residuos de cáscara de café en la metodología de superficie y enterrados disminuyeron la escorrentía en 10,2\% y $46 \%$, correspondientemente. (Moreno, Quizembe e Ibáñez, 2014)

Figura 4. Porcentaje de artículos publicados en relación con las aplicaciones de la cascarilla de café

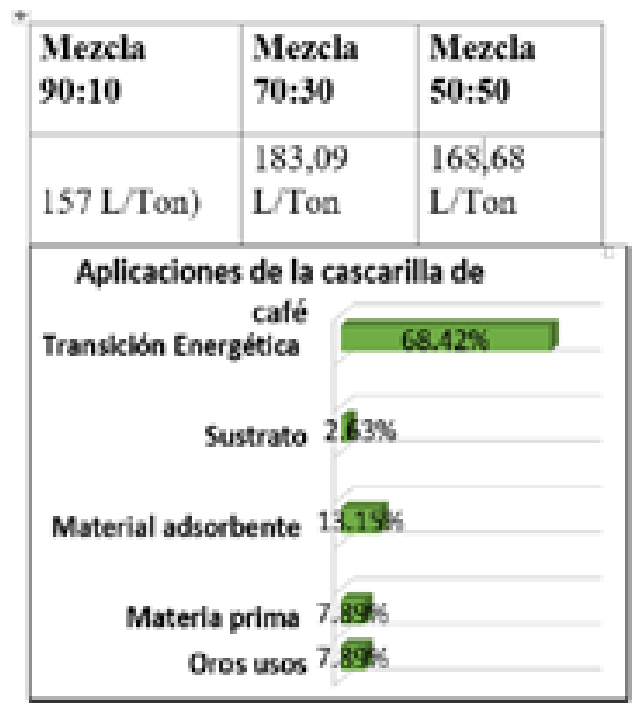

El porcentaje mayor de aplicación lo conlleva la transición energética de la cascarilla de café con un 68,42\% observado en la figura 4 , lo que da a relucir que se está empleando este residuo para 
la generación de biocombustibles sólidos, físicos y gaseosos; además de la generación de energía, como es posible observar en la figura 5 .

Figura 5. Porcentaje de las categorías con mayor aplicación energética de la cascarilla de café

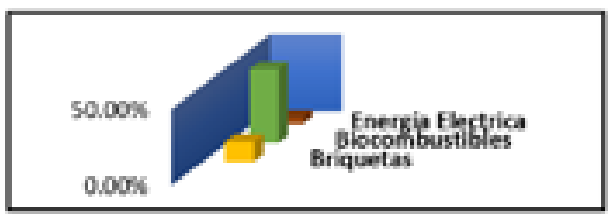

Tabla 4. Resultados de la comparación de mezclas realizadas por A. Álvarez Hincapié

\begin{tabular}{|l|l|l|}
\hline Mezcla 90:10 & Mezcla 70:30 & Mezcla 50:50 \\
\hline 157 L/Ton) & 183,09 L/Ton & 168,68 L/Ton \\
\hline
\end{tabular}

Fuente: Álvarez (2016).

Transición energética. La reconversión energética de la cascarilla de café se emplea mayoritariamente en la generación de biocombustibles. Moraima y Simbaña (2016) afirman que "la adecuación de biomasa y fabricación de biocombustibles sólidos, es una de las principales alternativas para satisfacer la demanda energética de las empresas; además de utilizar residuos agroindustriales es una fuente para la generación de empleo".
De acuerdo con las propiedades observadas en la tabla 3, "la cascarilla es considerada como materia prima apta para la elaboración de biocombustibles" (Arias y Meneses, 2016). La obtención de etanol de material lignocelulósico a partir de la cascarilla de café es una metodología beneficiosa, pues este residuo presenta un alto contenido de celulosa, sustrato de interés para la obtención de etanol, considerándose viable la generación a partir de este residuo agrícola (Vásquez, 2015). Así mismo, Tehrani, Aznar et al. (2015) obtuvieron tasas de producción de etanol entre 1,1 y 0,70 $g h-1 \mathrm{~kg}$-1 sin pretratamiento 2,7 y 2,3 gh-1 kg-1 y sustancia seca con pretratamiento suave respectivamente. Igualmente, Álvarez empleó el bagazo de caña de azúcar y la cascarilla de café, además de la mezcla de estas en porcentajes 90:10, 70:30, 50:50, respectivamente, para la producción de bioetanol, teniendo como premisa que el bagazo de caña de azúcar posee un alto rendimiento para la producción de etanol de 300-359 litros de etanol por tonelada de bagazo de caña de azúcar. Los valores obtenidos se pueden observar en la tabla 4. Con 183,09 L/Ton, la mezcla 70:30 resultó ser la mejor opción.

En Villanueva, teniendo en cuenta que al año 2014 se produjeron 734 to- 
neladas de café (MADR, 2014) y que se estima que por cada tonelada de frutos de café se producen alrededor de 0,18 toneladas de cascarilla de café (Álvarez, 2016), la cantidad generada fue de 132,12 toneladas de cascarilla en ese año. De la misma manera y tomando como premisa lo afirmado por Álvarez (2016), "por tonelada de cascarilla de café se producen entre 286,63 y 351,13 litros de etanol" en el municipio se producirían cerca de 46391,3 litros de comburente.

De otra forma, Dos Santos, Herrera et al. (2013) ejecutaron el proceso de ozonización de las cáscaras de café para la generación de hidrolizados que se emplearía en la producción de biogás mediante digestión anaeróbica, en la cual la producción más alta de metano fue de 36 $\mathrm{NmLCH} 4 / \mathrm{g} \mathrm{CH}$, en la que se recuperó $0,064 \mathrm{~kJ} / \mathrm{g} \mathrm{CH}$ de energía. Posteriormente, se agregó carbón activado en polvo debido a la presencia de compuestos tóxicos en el hidrolizado, lo cual mejoró la producción de biogás en $86 \mathrm{NmLCH} 4 /$ $\mathrm{gCH}$ y $0,58 \mathrm{~kJ} / \mathrm{gCH}$ en recuperación de energía, respectivamente. Teniendo en cuenta la recuperación de energía obtenida y el total de cáscaras producidas en el 2017 (2,7 millones de toneladas), si todos los residuos se transforman en metano el potencial de energía seria de 0,435 GWh (Dos Santos et al., 2018). En Villanueva el potencial estaría alrededor de 2,43X10-2GWh con 132,12 toneladas de cascarilla de café producidas en el 2014.

En otro orden de cosas, las briquetas desarrolladas a partir de cascaras de café son empleadas como fuente de combustible sostenible para labores domésticas de cocina. En este proceso se debe de tener en cuenta que se necesita de aglutinantes para de esta manera cohesionar los materiales; en este caso, Lubwama y Yiga (2017) usaron arcilla y almidón de yuca y la cascarilla de café con aglutinante de yuca presentó el valor de calentamiento más alto $21,9-23,0 \mathrm{MJ} / \mathrm{kg}$, mientras que el de arcilla 13,0 a 19,523 $\mathrm{MJ} / \mathrm{kg}$; esto es debido al tipo de aglutinante utilizado pues, en general, cambia propiedades físicas como su porcentaje calorífico y frente a la presencia de ceniza de $\mathrm{SiO} 2$ en la arcilla. Igualmente, Moraima y Simbaña (2016) destacan que "una briqueta de residuos lignocelulósicos en promedio puede superar las 3500 kcal. kg-1 en comparación de la leña que bordea los $2300 \mathrm{~K}$. cal. Kg-1". En la tabla 5, los resultados demostraron que la cascarilla de café compacta en un bloque sólido supera ampliamente la 
leña de eucalipto y de espino en cuanto a sus poderes caloríficos.

Tabla 5. Relación de poderes caloríficos de la briqueta de cascarilla de café, la leña de eucalipto y leña de espino

\begin{tabular}{|l|l|l|}
\hline $\begin{array}{l}\text { Briqueta de cascarilla } \\
\text { de café }\end{array}$ & $\begin{array}{l}\text { Leña } \\
\text { de eucalipto }\end{array}$ & $\begin{array}{l}\text { Leña de } \\
\text { espino }\end{array}$ \\
\hline 4162,31 k. cal. $\mathrm{kg}^{-1}$ & $\begin{array}{l}2173,90 \mathrm{k} . \mathrm{cal} . \\
\mathrm{kg}-1\end{array}$ & $\begin{array}{l}2236,24 \mathrm{k} . \\
\text { cal. } \mathrm{kg}-1\end{array}$ \\
\hline
\end{tabular}

Fuente: Moraima y Simbaña (2016).

Por otra parte, Dicovskiy, Pichardo et al. (2014) reemplazaron la leña con briquetas elaboradas de cascarilla de café en la producción de rosquillas de maíz, resultando que las briquetas constaban de un $25 \%$ más de poder energético que la leña de ripio de pino, además de que la generación de ceniza es muy poca en comparación con la leña. Otra ventaja de las briquetas de cascarilla de café es que no afecta la valoración sensorial de las rosquillas de maíz.

Por lo que se refiere a la generación de energía eléctrica, en la figura 5 se observa que es muy poca la aplicación realizada en esta temática, teniendo en cuenta el alto precio de las fuentes tradicionales de energía, la elevada demanda y los impactos ambientales por el uso de esta. D. Arenas Castellano (2009) propuso un gasificador en el cual el gas sea usado por un generador de 15 y $25 \mathrm{~kW}$ debido a que las fincas escogidas como objeto de estudio consumen mensualmente $3443 \mathrm{kWh}$. Considerando que la cascarilla de café cuenta con un porcentaje de humedad por debajo del $12 \%$ y su contenido de celulosa es mayor del $50 \%$, es un recurso atractivo para ser empleado en el proceso de gasificación con el objetivo de obtener energía eléctrica.

\section{Impactos ambientales y alterna- tivas para contrarrestar el inade- cuado manejo de subproductos del café}

En la vía húmeda de procesamiento del café el consumo de agua es alrededor de 4,2 L por kg de café cuando es utilizado el tanque de fermentación donde se elaboran las aguas mieles, las cuales al ser descargadas a los ríos incrementa la concentración de la materia orgánica, por lo que la acidez propia es afectada y el oxígeno disuelto disminuye. Esta problemática resulta en una disminución de biodiversidad en los cuerpos de agua. (Cárdenas, Arévalo y Vásquez, 2013)

De esta manera, Garay y Rivero (2014) consideran que "la depuración de 
las aguas residuales mediante la construcción de un biosistema o humedal con la presencia de macrófitas sembradas como Eichhornia crassipes en donde se almacenan las aguas del lavado del café es una metodología totalmente eficaz" a partir de los resultados obtenidos en la figura 6.

\section{Figura 6. Relación de DB05 y sólidos totales} en un humedal con macrófita Eichhornia crassipes.

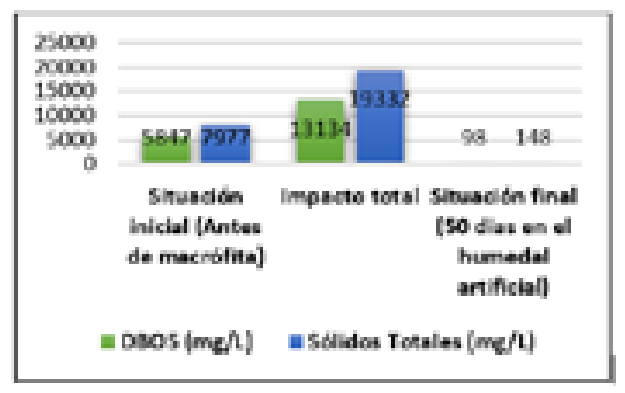

Fuente: Garay y Rivero (2014).

Al cabo de 50 días en el humedal artificial se alcanzó una reducción de $86,57 \%$ y $98,14 \%$ para la DBO5 y st, respectivamente (Garay y Rivero, 2014).

De la misma manera Pérez, Castillo et al. (2010) mencionan que la deforestación y pérdida de biodiversidad entendida como "una crisis de la civilización moderna, marcada por el deterioro de la naturaleza y una pésima calidad de vida", viene siendo propiciada por los monocultivos de café.
La erosión del suelo causado por este tipo de monocultivos también es de considerar, pues en áreas de alta precipitación pluvial el nitrógeno del suelo se pierde cerca de tres veces más en plantaciones sin sombra en concordancia con áreas con sombra. En relación con el vertimiento de aguas mieles, se afirma que

la disminución en los volúmenes de agua vertida equivale a una disminución en la contaminación generada por lo que actualmente se habla de "beneficios ecológicos" que favorecen el menor consumo de agua en las plantas de café como, por ejemplo, la recirculación del efluente en el propio beneficio y purificación de las aguas residuales. (Pérez et al., 2010)

Garay y Rivero (2014) consideran que para la obtención del café pergamino se necesitan $10 \mathrm{~L}$ de agua por kilogramo de café fresco; lo que quiere decir que se gasta $1 \mathrm{~m} 3$ de agua dulce por cada 1,7 quintales de café. Si se conoce que, en el municipio de Villanueva, en el 2014 se produjeron 7340 quintales de café por cosecha, respecto a la relación mencionada, se tendría una contaminación aproximada de 4317000 litros de agua dulce, atentando de esta manera con uno de los mayores recursos del municipio. 
En otros casos más impactantes, las cascarillas son arrojadas a las corrientes de agua, generando un aumento de principal importancia de la demanda bioquímica de oxígeno, sólidos totales, incremento en la temperatura del agua, proliferación de olores y, consecuentemente, pérdida de la calidad visual. Normalmente se presenta en épocas de cosecha en donde imposibilita el aprovechamiento del agua en acueductos, afectando la fauna y prohibiendo usos recreativos. (Garay y Rivero, 2014)

Por su parte, Pantoja, Rosero y Rosero (2016) afirman que, a pesar de las investigaciones realizadas por Cenicafe, aún existen zonas cafetaleras donde se utilizan más de 20 litros de agua limpia por cada kilogramo de café pergamino en el proceso de lavado y despulpe, así mismo, los subproductos: cascarilla, pulpa y mucilago son descargados en caños y ríos aledaños a fincas, generando graves afectaciones ambientales. Al respecto, Rodríguez y Zambrano (2010) reportan que "si se despulpara $5 \mathrm{~kg}$ de cereza de café y esta pulpa es arrojada directamente a las corrientes de agua, la contaminación sería similar o equivalente a los excrementos y orina generados por 6 personas en un día".

Del mismo modo, en Beyene et al. (2012) se evidenció un deterioro ecológico en los ríos aledaños, en los que son descargados los desechos orgánicos de las industrias de procesamiento de café. Los valores promedio de este análisis se pueden denotar en la tabla 6.

Tabla 6. Valores promedio de las características fisicoquímicas para los sitios de muestreos impactados por el procesamiento del café en relación con los sitios no impactados en el 2007 y 2008

\begin{tabular}{|l|l|l|l|l|}
\hline \multicolumn{3}{|l|}{ Muestro temporada húmeda (2007) } & \multicolumn{2}{l|}{ Muestro temporada seca (2008) } \\
\hline Parámetro & Sin impacto & Impactado & Sin impacto & Impactado \\
\hline DBO (mg/L) & 31 & 436 & 13 & 18 \\
\hline OD (mg/L) & 6,2 & 5,2 & 6,3 & 6,2 \\
\hline SDT (mg/L) & 117 & 170 & 101 & 120 \\
\hline SST (mg/L) & 192 & 598 & 152 & 158 \\
\hline pH & 7 & 6,2 & 7,2 & 7,1 \\
\hline Nitrato (mg/L) & 1,5 & 6,8 & 3,1 & 14 \\
\hline Amoniaco (mg/L) & 0,3 & 11 & 0,03 & 4 \\
\hline
\end{tabular}

Fuente: Beyene et al. (2012). 
En la tabla 6 se observa que entre los sitios sin impacto y con impacto por parte del procesamiento del café existe una notable diferencia como es el caso de la DBO y SST, prestando especial importancia a la disminución del oxígeno disuelto en la temporada húmeda, que determina que la presencia de materiales orgánicos oxidables de los residuos de café fueron causantes de la desoxigenación casi completa del sistema acuático, ocasionando que el impacto ejercido persistiera durante un tiempo excesivamente prolongado Beyene et al (2012). Esto significa que como en Villanueva no se realiza ningún tipo de tratamiento a la cascarilla de café, seguramente el recurso acuático se está viendo afectado de la misma manera y el impacto a la calidad del agua es considerablemente alto. Si se lleva al entorno estudiado en la temporada húmeda parámetros como la DBO, SDT y SST se aumentan en un $93 \%$, $59 \%$ y $75 \%$, respectivamente.

\section{Conclusiones}

1. La cascarilla de café es una fuente residual de biomasa con alto poder calorífico y con una eficiencia energética sustentable que brinda un sinnúmero de beneficios en cuanto al aprovechamiento del residuo agrícola para la generación de biocombustibles e, inclusive, como sustrato y como material adsorbente.

2. La caracterización fisicoquímica de la cascarilla de café es el parámetro determinante para la cantidad de generación de biocombustibles, sin embargo, este residuo presenta notables diferencias en todo el mundo debido a las condiciones de los suelos, del clima y de la preferencia del café cosechado, pues cabe destacar que son muchas las especies existentes de este fruto empleadas en diversos productos.

3. La elaboración de briquetas provee una alternativa sostenible para la utilización de este potencial energético en la generación de un comburente doméstico que proporciona más energía por unidad de volumen que el carbón y la leña.

4. El recurso hídrico es de los más afectados, pues las aguas residuales producto del beneficiado húmedo del café impactan agresivamente por ser aguas ácidas, alterando el agua dulce receptora con un $\mathrm{pH}$ alrededor de 5 .

5. Se determinó que en el municipio de Villanueva, La Guajira, en el 2014, se generaron cerca de 132,12 toneladas de cascarilla de café, las cuales no tuvieron utilización alguna, conta- 
minando cerca de 4317000 litros de agua dulce, desaprovechando la posibilidad de producir cerca de 46391,3 litros de etanol y un potencial energético de GWh.

6. El aprovechamiento de la cascarilla de café en el municipio permite reducir la contaminación del agua, atmósfera y suelo, además de disminuir la deforestación indiscriminada producto del uso de la leña.

\section{Referencias}

Acchar, W., Dultra, E. y Segadães, A. (2013). Untreated coffee husk ashes used as flux in ceramic tiles. Applied Clay Science, 75-76, 141-147.

Alhogbi, B. (2017). Potential of coffee husk biomass waste for the adsorption of $\mathrm{Pb}$ (II) ion from aqueous solutions. Sustainable Chemistry and Pharmacy, 6, 21-25.

Álvarez Hincapie, A. (2016). “Caracterización fisicoquímica de varios residuos agroindustriales y sus mezclas para la producción de biocombustibles". 85 .

Arenas Castellano, D. (2009). "Propuesta de diseño de un proceso para la generación de energía eléctrica a partir de los residuos de la porducción de café". 147.

Arias, R. y Meneses, J (2016). Caracterización residuos agroindustriales (cascarilla de arroz y cascarilla de café), como materia prima potencial para la obtención de bioetanol. Laboratorios de Química UNAN-Managua.
Barón, L. (2014). "Evaluación de la cascarilla de café como material adsorbente para la remoción de iones plomo $\mathrm{Pb} 2+$ presente en soluciones acuosas", p. 67.

Beyene, A., Kassahun, Y., Addis, T., Assefa, F., Amsalu, A., Legesse, W., Kloos, H. y Triest, L. (2012). The impact of traditional coffee processing on river water quality in Ethiopia and the urgency of adopting sound environmental practices. Environ. Monit. Assess., 184(11), 7053-7063.

Cárdenas Arévalo, J. y Vásquez López, J. (2013). "Análisis del ciclo de vida del procesamiento y la distribución del café del beneficio ecológico en la finca Juancito y convencional en la finca La Montaña, Francisco Morazán, Honduras”. p. 22.

Carrillo, M., Zavala, D., Alvarado, K. Morales, S. y Bautista, P. (2011). Obtención de biomasa a partir de cáscara de cafe. Revista Académica de Investigación, 14.

Coffea (2010). La cascarilla de cafe como materia prima para elaborar papel. Universidad Nacional Autónoma de México.

Conesa, J., Sánchez, N., Garrido, M. y Casas, J. (2016). Semivolatile and Volatile Compound Evolution during Pyrolysis and Combustion of Colombian Coffee Husk. Energy and Fuels, 30(10), 7827-7833.

Cubides-Hernández, A. y Montaña-Martínez, C. (2017). Evaluación del impacto ambiental generado por los residuos peligrosos en el sector agrícola en la vereda de Chámeza, municipio de Nobsa, Boyacá. Sogamoso, Boyacá: Universidad Nacional Abierta y a Distancia.

De Oliveira, J., Da Silva, J., Graciosa Pereira, E., Oliveira Filho, D. y Rizzo Carvalho, D. 
(2013). Characterization and mapping of waste from coffee and eucalyptus production in Brazil for thermochemical conversion of energy via gasification. Renewable and Sustainable Energy Reviews, 21, 5258.

De Oliveira, J., Da Silva, J., Martins, M., Pereira, E. y Da Conceição, M., Trindade Bezerra e Oliveira (2018). Gasification of waste from coffee and eucalyptus production as an alternative source of bioenergy in Brazil. Sustainable Energy Technologies and Assessments, 27, 159-166.

Dicovskiy, L., Pichardo, C., Rodríguez Celedón, A., Martínez Centeno, B. y Rodríguez Espinoza, K. (2014). Validación de briquetas elaboradas con cascarilla de café para el remplazo de leña en la producción de rosquillas de maíz. Validation briquettes made whit deseeded coffee for replacement of wood in the production of corn rosquillas. El Higo, 4, 10-19.

Dos santos, L., Herrera Adarme, O., Lobo Baeta, B., Alves Gurgel, L. y Francisco de Aquino, S. (2018). Production of biogas (methane and hydrogen) from anaerobic digestion of hemicellulosic hydrolysate generated in the oxidative pretreatment of coffee husks. Bioresour Technol., 263, 601-612.

Franca, S. y Oliveira, L. (2009). Coffee Processing Solid Wastes: Current Uses and Future Perspectives.

Garay Roman, J. y Rivero Méndez, J. (2014). Biosistema para purificar aguas residuales del beneficio húmedo de café, distrito $\mathrm{La}$ Coipa, departamento de Cajamarca, 11(1), 43-50.
Granados, C. (1994), "El impacto ambiental del café en la historia costarricense". p. 34.

Louzada, L., Fonseca, L. y De Oliveira, J. (2016). Evaluation of the toxic potential of coffee wastewater on seeds, roots and meristematic cells of Lactuca sativa L. Ecotoxicology and Environmental Safety, 133, 366-372.

Lubwama, M. y Yiga, V. (2017). Characteristics of briquettes developed from rice and coffee husks for domestic cooking applications in Uganda. Renewable Energy, 118, 43-55.

Manals-cutiño, E., Salas-tort, D. y Penedo-Medina, M. (2018). Caracterización de la biomasa vegetal "cascarilla de café". XXXVIII(1), 198-213.

Mera Aguas, M. y Simbaña Villarreal, E. (2016). Evaluación de la capacidad calorífica de biocombustible sólido a partir de residuos lignocelulósicos de café. Axioma, $35-41$.

Ministerio de Agricultura y Desarrollo Rural (MADR) (2014). Principales cultivos por área sembrada en el año 2014. Cultivo.

Moreno-Ramón, H., Quizembe, S. y Ibáñez-Asensio, S. (2014). Coffee husk mulch on soil erosion and runoff: Experiences under rainfall simulation experiment. Solid Earth, 5(2), 851-862.

Municipio de Villanueva (2000). Esquema de ordenamiento territorial, análisis y síntesis territorial. Parte I.

Oliveira, L., Franca, A., Alves, T. y Rocha, S. (2008). Evaluation of untreated coffee husks as potential biosorbents for treatment of dye contaminated waters. Journal of Hazardous Materials., 155(3), 507-512. 
Oliveira, W., Franca, A., Oliveira, L. y Rocha, S. (2008). Untreated coffee husks as biosorbents for the removal of heavy metals from aqueous solutions. Journal of Hazardous Materials, 152(3), 1073-1081.

Pantoja-Figueroa, C., Rosero-Rodríguez, A. y Rosero Rodríguez, L. (2016). "Los residuos de la poscosecha del café 1." San Juan de Pasto, pp. 1-78. of Lactuca sativa L.". Ecotoxicology and Environmental Safety, 133, 366-372.

Pérez Díaz, N., Castillo Ramos, R., Carballo Abreu, L. y Veliz Gutiérrez, J. (2010). "Impacto ambiental en el cultivo y procesamiento del café y su repercusión social”. 22.

Rodríguez, N. y Zambrano, D. (2010). Los subproductos del café: fuente de energía renovable. Avances Técnicos Cenicafé, 3, 8 .

Romero, R. y Mamani, R. (2013). Obtención de biogás como fuente de energía renovable a partir de los subproductos del café. Biomass and Bioenergy, 15, 241-252.
Roque, H., Medrano, J. y Sierra, J. (2013). Aprovechamiento de la cascarilla de café en la elaboración de materiales de construcción. El Higo. Revista de Ciencia y Tecnología, 3, 7-9.

Sinergia (2006). Producción respetuosa en viticultura. Impactos ambientales en agricultura. Proyecto Life Sinergia, 1-11.

Tehrani, N., Aznar, J. y Kiros, Y. (2015). Coffee extract residue for production of ethanol and activated carbons. Journal of Cleaner Production, 91, 64-70.

Toscano, L. (2009). ““Análisis de los parámetros y Selección de hornos para la combustión de biomasa' (aplicación a biomasas locales típicas)", 181.

Vázquez Morales, O. (2015). "Etanol lignocelulósico, a partir de cascarilla de café, por medio de hidrólisis química-enzimática y fermentación". 150. 